\title{
PURPOSE OF THE SYMPOSIUM
}

\author{
B. N. Charles ${ }^{1}$ \\ Aerospace Corporation, El Segundo, Calif.
}

Broadly stated, the purpose of this symposium is to facilitate the free exchange of scientific information about the upper atmosphere. This conforms to one of the prime objectives of the American Meteorological Society-the "development and dissemination of knowledge of meteorology in all its phases and applications." A wealth of scientific experience has proven that such exchanges inspire new findings, generate new concepts, and refresh and stimulate the seekers of new knowledge and those who apply such knowledge in the service of society.

But in an era of such proliferation of scientific information as to cause concern, why this subject, at this time, and why the effort to assemble such a distinguished roster of participants?

One aspect of the remarkable rapid advances in aerospace technology that we have been privileged to witness during the past decade has been the recurring need to design and operate vehicles in environments that are inadequately understood. The upper atmosphere is one such environment, and the paucity of needed design information has undoubtedly levied substantial penalties in decreased systems performance, increased costs, and delayed implementation of innovations that could contribute significantly to a broad spectrum of social goals, including those of scientific investigation.

For a more detailed exposition of these matters, I commend to you the article by Dr. Sidney Teweles, in the Bulletin of the American Meteorological Society of April 1967, in which the opinion is expressed that increased coordination of scientific and technological effort is needed in upper atmospheric investigations.

${ }^{1}$ Chairman, AMS Committee on Atmospheric Problems of Aerospace Vehicles.
These considerations prompted the initiative of the AMS Committee on Atmospheric Problems of Aerospace Vehicles in arranging this symposium. Our specific purpose is the convening of a cross-disciplinary seminar at which scientists and technologists can exchange information and views, and identify those areas of mutual interest wherein increased coordination of effort might accelerate progress in understanding of the atmosphere above $70 \mathrm{~km}$, and in application of such new technical capital to pragmatic purposes. In recent years we have witnessed a convergence of interest of and effort by meteorologists and aeronomers, in studies of the upper atmosphere. In a sense, we are here suggesting that a similar community of interest exists for scientists and engineers concerned with the same medium, and that a similar convergence of interest and effort may also be rewarding.

The approach adopted by the Program Committee, under Dr. Teweles' leadership, begins with comprehensive reviews of the state of knowledge of the mesosphere and lower thermosphere. These are to be supplemented by panel discussions intended to illuminate the more significant deficiencies in knowledge, considering scientific and engineering needs, and which, hopefully, will further identify specific avenues for increased coordination of investigative efforts.

It is my pleasure and privilege to express appreciation to the distinguished contributors to the program, to the program committee that labored so effectively, to the National Science Foundation for enabling the participation of our colleagues from overseas, to the National Aeronautics and Space Administration for extending support for the publication of these proceedings, and to those in the audience for valued participation. 